\title{
ZERO-ORDER MEHLER-FOCK TRANSFORM AND SOBOLEV-TYPE SPACE
}

\author{
AKhilesh Prasad, U. K. MAndal And S. K. VERma
}

Abstract. The present paper is devoted to the study of the Mehler-Fock transform with index as the Legendre function of first kind. Continuity property of the Mehler-fock transform on the test function spaces $\Lambda_{\alpha}$ and $\mathscr{G}_{\alpha}$ is given. Moreover pseudo-differential operator (p.d.o.) with symbol $\sigma(x, \tau) \in S^{m}$ in terms of Mehler-Fock transform is defined and also its continuity property from test function space $\mathscr{G}_{\alpha}$ into $\Lambda_{\alpha}$ is shown. The Mehler-Fock potential (MF-potential) $\mathscr{P}_{\sigma}^{s}$ is defined on $\mathscr{G}_{\alpha}(I)$ space and it is extended to the space of distribution. Also some properties of MF-potential are discussed. At the end Sobolev type space $V^{s, p}(I)$ is defined and it is shown that MF-potential is an isometry of $V^{s, p}(I)$.

Mathematics subject classification (2010): 44A20, 44A15, 46E35, $35 \mathrm{~S} 05$.

Keywords and phrases: Mehler-Fock transform, Sobolev type space, convolution, pseudo-differential operator.

\section{REFERENCES}

[1] A. Erdélyi, W. Magnus, F. Oberhettinger and F. Tricomi, Higher Transcendental Functions, Vol. 1, McGraw-Hill, New York, 1953.

[2] V. A. Fock, On the representation of an arbitrary function by an integral involving Legendre's function with a complex index, Dokl. Akad. Nauk SSSR, 39, (1943), 279-283 (in Russian).

[3] H. J. Glaes Ke AND A. Hess, A convolution connected with the Kontorovich-Lebedev transform, Math. Z. 193, 1 (1986), 67-78.

[4] H. J. Glaes Ke AND A. Hess, On the convolution theorem of the Mehler-Fock transform for a class of generalized functions (I), Math. Nachr. 131, 1 (1987), 107-117.

[5] H. J. Glaeske, A. P. Prudnikov And K. A. SkóRnik, Operational Calculus and Related Topics, Chapman \& Hall/CRC, Boca Raton, FL, 2006.

[6] B. J. GonzÁlez And E. R. NegRín, Mehler-Fock transforms of generalized functions via the method of adjoints, Proc. Amer. Math. Soc. 125, 11 (1997), 3243-3253.

[7] Y. E. Gutiérrez-Tovar And J. M. R. MÉndez-PÉrez, A convolution for the HankelKontorovich-Lebedev transformation, Math. Nachr. 281, 11 (2008), 1566-1581.

[8] N. HAYEK AND B. J. GONZÁLEZ, On the Mehler-Fock transform of generalized functions, Bull. Soc. Roy. Sci. Liége 61, 3-4 (1992), 315-327.

[9] L. Hörmander, Pseudo-differential operators, Comm. Pure Appl. Math. 18, 3 (1965), 501-517.

[10] J. J. Kohn And L. NiRenberg, An algebra of pseudo-differential operators, Comm. Pure Appl. Math. 18, 1-2 (1965), 269-305.

[11] N. N. Lebedev, Special Functions and Their Applications, Eaglewood Cliffs, N. J., Prentice-Hall, 1965.

[12] N. N. Lebedev, The Parseval theorem for the Mehler-Fock integral transform, Dokl. Akad. Nauk SSSR, 68, 3 (1949), 445-448 (in Russian).

[13] F. G. MEHLER, Ueber eine mit den kugel-und cylinderfunctionen verwandte function und ihre anwendung in der theorie der elektricitatsvertheilung, Math. Anal. 18 (1881), 161-194.

[14] L. Nirenberg, Pseudo-Differential Operators, Springer, (2011).

[15] F. W. J. Olver, Asymptotics and Special Functions, Academic Press, New York, 1974. 
[16] F. W. J. Olver, D. W. Lozier, R. F. Boisvert and C. W. Clark (Eds.), NiST Handbook of Mathematical Functions, Cambridge University Press, Cambridge, 2010.

[17] M. J. OLEVSKIJ, On the representation of an arbitrary function in the form of an integral with a kernel containing a hypergeometric function, Dokl. Akad. Nauk SSSR 69, 1 (1949), 11-14 (in Russian).

[18] A. Passian, S. Koucheckian, S. B. Yakubovich and T. Thundat, Properties of index transforms in modeling of nanostructures and plasmonic systems, J. Math. Phy. 51, 2 (2010): 023518, 30 pp.

[19] R. S. Pathak And P. K. PANdey, A class of pseudo-differential operators associated with Bessel operators, J. Math. Anal. Appl. 196, 2 (1995), 736-747.

[20] R. S. PathaK And P. K. PAndey, Sobolev type spaces associated with Bessel operator, J. Math. Anal. Appl. 215, 1 (1997), 95-111.

[21] R. S. PATHAK AND S. K. Upadhyay, Pseudo-differential operators involving Hankel transforms, J. Math. Anal. Appl. 213, 1 (1997), 133-147.

[22] A. PRASAD AND U. K. MANDAl, Boundedness of pseudo-differential operators involving Kontorovich-Lebedev transform, Integral Transforms Spec. Funct. 28, 4 (2017), 300-314.

[23] A. PRASAD, S. K. Verma AND U. K. Mandal, The convolution for zero-order Mehler-Fock transform and pseudo-differential operator, Integral Transforms Spec. Funct. 29, 3 (2018), 189-206.

[24] L. Rodino, Linear Partial Differential Operators in Gevrey Spaces, World Scientific, Singapore, 1993.

[25] M. M. Rodrigues, N. VieIRA AND S. YAKUbovich, A convolution operator related to the generalized Mehler-Fock and Kontorovich-Lebedev transforms, Results Math. 63, 1-2 (2013), 511-528.

[26] N. B. Salem AND A. Dachraoui, Pseudo-differential operators associated with the Jacobi differential operator, J. Math. Anal. Appl. 220, 1 (1998), 365-381.

[27] N. B. Salem AND A. Dachraoui, Sobolev type spaces associated with the Jacobi differential operators, Integral Transforms Spec. Funct. 9, 3 (2000), 163-184.

[28] I. N. SNEdDon, The Use of Integral Transforms, McGraw-Hill Book Company, New York, 1972.

[29] H. M. SRivastava, B. J. GonZÁLez And E. R. Negrín, New Lp-boundedness properties for the Kontorovich-Lebedev and Mehler-Fock transforms, Integral Transforms Spec. Funct. 27, 10 (2016), 835-845.

[30] N. SRIVAStaVA, On dual integral equations associated with Mehler-Fock transform, J. Indian Acad. Math. 13, 2 (1991), 115-122.

[31] N. YA. VILENKIN, The matrix elements of irreducible unitary representations of a group of Lobachevsky space motions and the generalized Fock-Mehler transformations, Dokl. Akad. Nauk SSSR 118, 2 (1958), 219-222 (in Russian).

[32] M. W. Wong, An Introduction to Pseudo-Differential Operators, 3rd ed., World Scientific, Singapore, 2014.

[33] S. B. Yakubovich, Index Transforms, World Scientific, Singapore, 1996.

[34] S. B. Yakubovich And Y. F. Luchko, The Hypergeometric Approach to Integral Transforms and Convolutions, vol. 287, Kluwer Academic Publishers, Dordrecht, 1994. 